Arq. Bras. Med. Vet. Zootec., v.65, n.2, p.610-618, 2013

\title{
Qualidade de ovos e resistência óssea de poedeiras alimentadas com minerais orgânicos
}

\author{
[Quality of eggs and bone strength of layers fed organic minerals ] \\ J.K. Nunes ${ }^{1}$, V.L. Santos ${ }^{1}$, P. Rossi ${ }^{1}$, M.A. Anciuti ${ }^{2}$, F. Rutz ${ }^{1}$, J.C. Maier ${ }^{1}$, J.G.C. Silva ${ }^{1}$ \\ ${ }^{1}$ Universidade Federal de Pelotas - Capão do Leão, RS \\ ${ }^{2}$ Instituto Federal Sul Rio-Grandense Campus Pelotas -Visconde da Graça - Pelotas, RS
}

\section{RESUMO}

\begin{abstract}
Avaliou-se o efeito da utilização de níveis crescente de minerais orgânicos sobre a qualidade externa e interna de ovos e a resistência de ossos da tíbia de poedeiras semi-pesadas. Foram utilizadas 256 aves, com idade inicial de 30 semanas, distribuídas em 64 gaiolas perfazendo quatro aves por gaiola em delineamento inteiramente ao acaso. Os tratamentos consistiram da inclusão de minerais inorgânicos ou níveis crescentes de orgânicos na dieta basal das poedeiras, resultando em quatro tratamentos. Foram avaliados peso do ovo (g), gravidade específica, peso $(\mathrm{g})$ e espessura $(\mathrm{mm})$ da casca, altura do albúmen $(\mathrm{mm})$, unidade Haugh, peso da gema $(\mathrm{g})$ e do albúmen $(\mathrm{g})$ e resistência óssea $(\mathrm{kg})$. Houve diferença significativa $(\mathrm{P}>0,05)$ entre as médias para peso do ovo e do albúmen. $\mathrm{O}$ fornecimento de dietas suplementadas com minerais orgânicos, em nível intermediário resultou na produção de ovos mais pesados, com maior peso de albúmen, e na manutenção das demais características de qualidade externa e interna dos ovos e de resistência óssea.
\end{abstract}

Palavras-chave: poedeira, peso do albúmen, peso do ovo

\begin{abstract}
The effect of increasing levels of organic minerals on the external and internal quality of eggs and tibia bone strength of brown-egg laying hens was studied. A total of 256, 30-week old was equally distributed in 64 cages in a total of four birds per cage in a completely randomized experimental design. The dietary treatments consisted of inorganic or increasing levels of organic minerals in the diets of laying hens, resulting in four treatments. Egg weight ( $g$ ), specific gravity, shell weight and thickness (mm), albumen height $(\mathrm{mm})$, Haugh unit, yolk weight and albumen $(\mathrm{g})$ and bone strength $(\mathrm{kg})$ were evaluated. A significant difference $(P \leq 0.05)$ among treatment means for egg weight and albumen was found. Intermediate inclusion levels of organic minerals resulted in the production of heavier eggs, with higher albumen weight and maintenance of the other characteristics of internal and external quality of eggs and bone strength.
\end{abstract}

Keywords: laying hen, albumen weight, egg weight

\section{INTRODUÇÃO}

Os minerais estão presentes, em diversas concentrações, nos ingredientes usualmente empregados nas formulações das dietas e na constituição dos tecidos animais, e representam cerca de 3 a $4 \%$ do peso vivo das aves (Saldanha et al., 2009). Portanto, a suplementação mineral na alimentação é de extrema importância para o desenvolvimento e a manutenção dos animais (Polli, 2002). As primeiras pesquisas sobre as fontes de minerais passíveis de serem utilizadas nas dietas remetem à década de 50; neste período, os esforços eram destinados à utilização da suplementação mineral como solução para problemas ósseos e de desempenho produtivo das aves (Araujo et al., 2008).

Recebido em 9 de setembro de 2011

Aceito em 20 de julho de 2012

E-mail: julianaklug@yahoo.com.br 
Com relação à quantidade de suplementação, os minerais são classificados como macro ou microelementos, ou seja, quando adicionados às dietas em quantidades superiores ou inferiores a $70 \mathrm{mg} k \mathrm{~kg}$ de peso vivo do animal (Bondei, 1987). Entre os microminerais, o ferro e o selênio participam de inúmeras reações de síntese de compostos do organismo animal, como da hemoglobina e dos hormônios tireoidianos, além de atuarem na manutenção da integridade das membranas biológicas (MacDowell, 1992). O zinco, o manganês e o cobre estão associados ao crescimento e ao desenvolvimento do tecido ósseo.

Hoje, além dos minerais inorgânicos, os nutricionistas contam com as fontes orgânicas. Segundo Veiga e Cardoso (2005), os microelementos, ao serem ofertados na forma de um complexo orgânico ou quelatos, proteinatos ou polissacarídeos, apresentam o seu valor biológico otimizado. Uma das principais características dos minerais orgânicos é o fato de serem mais biodisponíveis, o que representa melhor aproveitamento, sendo, portanto, menos excretáveis pelos animais, proporcionando a estes melhores condições para expressão de seu genótipo (Rutz e Murphy, 2009).

Kratzer e Vohra (1986) afirmaram que os minerais orgânicos geralmente utilizam as vias de absorção das moléculas que os ligam, o que faz com que não tenham problemas de interações com outros minerais, resultando em absorção superior quando comparados aos inorgânicos. Para Reddy et al. (1992) e Clydesdale (1998), o ligante forma um composto solúvel com o mineral, sendo, com isso, melhor absorvido pela mucosa intestinal e, consequentemente, mais biodisponível. De acordo com Junqueira (2008), a biodisponibilidade de minerais orgânicos pode ultrapassar $90 \%$, o que poderá resultar em maior produção de ovos, casca mais resistente, menor mortalidade e redução dos efeitos do estresse (Sechinatto, 2003), visto que, durante a ação de fatores estressantes, seja de forma aguda ou crônica, vários minerais são mobilizados, sendo, por conseguinte, excretados em maior quantidade, podendo levar a perdas econômicas e à contaminação do meio ambiente (Malleto, 1997).

Como todos os animais, as poedeiras precisam ter suas necessidades nutricionais atendidas diariamente, como aporte para a manutenção de sua produtividade. Assim sendo, as exigências de nutrientes variam com a idade, a linhagem, a taxa de produção, o tamanho do ovo e o clima. Estudos realizados por Kienholz (1992), Xavier et al. (2004), Rutz et al. (2005) e Brito et al. (2006) apontam a suplementação mineral atuando positivamente na resistência óssea, na excreção minerálica, no desempenho produtivo e na qualidade dos ovos.

O trabalho desenvolvido teve por objetivo a avaliação da substituição dos minerais inorgânicos por orgânicos sobre a qualidade externa e interna de ovos e a resistência óssea de poedeiras semipesadas.

\section{MATERIAL E MÉTODOS}

O experimento foi aprovado pela Comissão de Ética e Experimentação Animal da Universidade Federal de Pelotas e está protocolado sob número 23110.000618/2009-74.

Durante 280 dias, divididos em 10 ciclos, foram utilizadas 256 poedeiras semipesadas da linhagem Hisex Brown, com idade inicial de 30 semanas. As aves foram alojadas em 64 gaiolas de um aviário do tipo dark house, e a unidade experimental foi representada pela gaiola com quatro poedeiras alojadas. A densidade de alojamento respeitou o protocolo de bem-estar para aves poedeiras (União..., 2008), que é de $450 \mathrm{~cm}^{2} /$ ave. O fornecimento de ração e de água foi à vontade, sendo que, para a alimentação, foram utilizados comedouros do tipo calha aberta, dispostos na frente das gaiolas. Já a água foi disponibilizada por meio de bebedouros tipo nipple, de maneira que as poedeiras de cada gaiola tivessem acesso a dois bebedouros.

As poedeiras foram submetidas a um fotoperíodo de $16 \mathrm{~h}$ de luz diária. A temperatura e a umidade interna do aviário foram registradas por um termo-higrômetro de mínima e máxima, tendo oscilado, durante o período experimental, entre $18,0^{\circ} \mathrm{C}$ e $25,0^{\circ} \mathrm{C}$ e entre $50 \%$ e $65 \%$, respectivamente.

Os tratamentos (Tab. 1 e 2) consistiram da inclusão de minerais inorgânicos e porcentagens de minerais orgânicos na dieta basal das poedeiras, resultando em quatro tratamentos, em que $\mathrm{T}_{1}$ : dieta basal $+0,3 \mathrm{ppm}$ de selenito de $\mathrm{Na}+$ 
60ppm óxido de $\mathrm{Zn}+60 \mathrm{ppm}$ monóxido de $\mathrm{Mn}+30 \mathrm{ppm}$ de sulfato $\mathrm{Fe}+0,5 \mathrm{ppm}$ de iodato de $\mathrm{Ca}+6,0 \mathrm{ppm}$ de sulfato de $\mathrm{Cu} ; \mathrm{T}_{2}$ : dieta basal $+0,1 \mathrm{ppm}$ de Se levedura $+20 \mathrm{ppm}$ de proteinato de $\mathrm{Zn}+20 \mathrm{ppm}$ de proteinato de $\mathrm{Mn}+10 \mathrm{ppm}$ de proteinato de $\mathrm{Fe}+2,0 \mathrm{ppm}$ de proteinato de $\mathrm{Cu}+0,5 \mathrm{ppm}$ de iodato de $\mathrm{Ca} ; \mathrm{T}_{3}$ : dieta basal $+0,2 \mathrm{ppm}$ de Se levedura $+40 \mathrm{ppm}$ de proteinato de $\mathrm{Zn}+40 \mathrm{ppm}$ de proteinato de $\mathrm{Mn}+20 \mathrm{ppm}$ de proteinato de $\mathrm{Fe}+4,0 \mathrm{ppm}$ de proteinato de $\mathrm{Cu}+0,5$ pm de iodato de $\mathrm{Ca} ; \mathrm{T}_{4}$ : dieta basal $+0,3$ ppm de Se levedura $+60 \mathrm{ppm}$ de proteinato de $\mathrm{Zn}+60 \mathrm{ppm}$ de proteinato de $\mathrm{Mn}+30 \mathrm{ppm}$ de proteinato de $\mathrm{Fe}+6,0 \mathrm{ppm}$ de proteinato de $\mathrm{Cu}+0,5 \mathrm{ppm}$ de iodato de $\mathrm{Ca}$. As dietas formuladas eram isocalóricas, isocálcicas, isofosfóricas e isosódicas, variando os níveis em função do atendimento das exigências nutricionais, de acordo com a idade das aves.

Tabela 1. Composição das dietas experimentais fornecidas às poedeiras, no período de 30 a 45 semanas de idade

\begin{tabular}{|c|c|c|c|c|}
\hline Ingrediente, \% & $\mathrm{T}_{1}$ & $\mathrm{~T}_{2}$ & $\mathrm{~T}_{3}$ & $\mathrm{~T}_{4}$ \\
\hline Milho & 62,87 & 62,87 & 62,87 & 62,87 \\
\hline Farelo de soja & 24,80 & 24,80 & 24,80 & 24,80 \\
\hline Farelo de trigo & 0,81 & 0,81 & 0,81 & 0,81 \\
\hline Farinha de ostra & 8,00 & 8,00 & 8,00 & 8,00 \\
\hline Sal iodado & 0,37 & 0,37 & 0,37 & 0,37 \\
\hline Suplemento mineral inorgânico ${ }^{1}$ & 0,05 & - & - & - \\
\hline $\begin{array}{l}\text { Suplemento mineral e vitamínico e } \\
\text { aminoácidos }^{2}\end{array}$ & 3,00 & 3,00 & 3,00 & 3,00 \\
\hline Caulim & 0,1000 & 0,1048 & 0,0596 & 0,0144 \\
\hline Selênio levedura ${ }^{3}$ & - & 0,0100 & 0,0200 & 0,0300 \\
\hline Proteinato de zinco ${ }^{4}$ & - & 0,0133 & 0,0266 & 0,0399 \\
\hline Proteinato de manganês ${ }^{5}$ & - & 0,0133 & 0,0266 & 0,0399 \\
\hline Proteinato de ferro ${ }^{6}$ & - & 0,0066 & 0,0132 & 0,0198 \\
\hline Proteinato de cobre ${ }^{7}$ & - & 0,0020 & 0,0040 & 0,0060 \\
\hline Total & 100,00 & 100,00 & 100,00 & 100,00 \\
\hline \multicolumn{5}{|l|}{ Nível nutricional calculado } \\
\hline Energia metabolizável (kcal/kg) & 2720 & 2720 & 2720 & 2720 \\
\hline Proteína bruta $(\%)$ & 16,600 & 16,600 & 16,600 & 16,600 \\
\hline Cálcio $(\%)$ & 3,800 & 3,800 & 3,800 & 3,800 \\
\hline Fósforo disponível (\%) & 0,383 & 0,383 & 0,383 & 0,383 \\
\hline Metionina total (\%) & 0,353 & 0,353 & 0,353 & 0,353 \\
\hline Lisina total $(\%)$ & 0,893 & 0,893 & 0,893 & 0,893 \\
\hline Extrato etéreo (\%) & 2,729 & 2,729 & 2,729 & 2,729 \\
\hline Fibra bruta (\%) & 2,980 & 2,980 & 2,980 & 2,980 \\
\hline Sódio total (\%) & 0,180 & 0,180 & 0,180 & 0,180 \\
\hline
\end{tabular}

T1: dieta basal + 0,3ppm de selenito de $\mathrm{Na}+60 \mathrm{ppm}$ óxido de $\mathrm{Zn}+60 \mathrm{ppm}$ monóxido de $\mathrm{Mn}+30 \mathrm{ppm}$ de sulfato Fe $+0,5 \mathrm{ppm}$ de iodato de $\mathrm{Ca}+6,0 \mathrm{ppm}$ de sulfato de $\mathrm{Cu}$; T2: dieta basal $+0,1 \mathrm{ppm}$ de Se levedura $+20 \mathrm{ppm}$ de proteinato de $\mathrm{Zn}+20 \mathrm{ppm}$ de proteinato de $\mathrm{Mn}+10 \mathrm{ppm}$ de proteinato de $\mathrm{Fe}+2,0 \mathrm{ppm}$ de proteinato de $\mathrm{Cu}+0,5 \mathrm{ppm}$ de iodato de $\mathrm{Ca}$; $\mathrm{T} 3:$ dieta basal $+0,2 \mathrm{ppm}$ de Se levedura $+40 \mathrm{ppm}$ de proteinato de $\mathrm{Zn}+40 \mathrm{ppm}$ de proteinato de $\mathrm{Mn}+20 \mathrm{ppm}$ de proteinato de $\mathrm{Fe}+4,0 \mathrm{ppm}$ de proteinato de $\mathrm{Cu}+0,5 \mathrm{ppm}$ de iodato de $\mathrm{Ca}$; T4: dieta basal $+0,3 \mathrm{ppm}$ de Se levedura $+60 \mathrm{ppm}$ de proteinato de $\mathrm{Zn}+60 \mathrm{ppm}$ de proteinato de $\mathrm{Mn}$ +30 ppm de proteinato de $\mathrm{Fe}+6,0 \mathrm{ppm}$ de proteinato de $\mathrm{Cu}+0,5 \mathrm{ppm}$ de iodato de $\mathrm{Ca}$.

Garantia por kg do produto:

${ }^{1}$ Suplemento mineral inorgânico (Alltech Agroindustrial do Brasil): monóxido de Mn: 140000mg, óxido de Zn: 100000mg, sulfato ferroso: $120000 \mathrm{mg}$, sulfato de $\mathrm{Cu}: 200000 \mathrm{mg}$, iodato de Ca: $1400 \mathrm{mg}$, selenito de $\mathrm{Na}: 700 \mathrm{mg}$.

${ }^{2}$ Suplemento mineral, vitamínico e aminoácido: Ca: 269g, P: 94g, Mn: 2334mg, Zn: 1667mg, Fe: 2000mg, Cu: 334mg, I: 12mg, Se: 10,2mg, vit. A: 334000 UI, vit. D3: 67000 UI, vit. E: 234mg, vit. K3: 50mg, vit. B1: 54mg, vit. B2 147mg, vit. B6: 100g, vit. B12: 400mcg, niacina: $867 \mathrm{mg}$, ácido fólico: $24 \mathrm{mg}$, ácido pantotênico: $334 \mathrm{mg}$, metionina: $34 \mathrm{~g}$.

${ }^{3}$ Suplemento mineral orgânico ${ }^{1}:$ Se levedura: $1000 \mathrm{mg} / \mathrm{kg}$

${ }^{4}$ Suplemento mineral orgânico ${ }^{1}$ : proteinato de $\mathrm{Zn}: 15 \%$.

${ }^{5}$ Suplemento mineral orgânico ${ }^{1}$ : proteinato de $\mathrm{Mn}: 150000 \mathrm{mg} / \mathrm{kg}$.

${ }^{6}$ Suplemento mineral orgânico ${ }^{1}$ : proteinato de Fe: $150000 \mathrm{~g}$.

${ }^{7}$ Suplemento mineral orgânico ${ }^{1}$ : proteinato de $\mathrm{Cu}: 100000 \mathrm{~g}$. 
Qualidade de ovos...

Tabela 2. Composição das dietas experimentais fornecidas às poedeiras, no período de 46 a 70 semanas de idade

\begin{tabular}{|c|c|c|c|c|}
\hline Ingrediente, $\%$ & $\mathrm{~T}_{1}$ & $\mathrm{~T}_{2}$ & $\mathrm{~T}_{3}$ & $\mathrm{~T}_{4}$ \\
\hline Milho & 63,13 & 63,10 & 63,13 & 63,13 \\
\hline Farelo de soja & 23,20 & 23,20 & 23,20 & 23,20 \\
\hline Farelo de trigo & 1,45 & 1,45 & 1,45 & 1,45 \\
\hline Farinha de ostra & 8,70 & 8,70 & 8,70 & 8,70 \\
\hline Sal iodado & 0,37 & 0,37 & 0,37 & 0,37 \\
\hline Suplemento mineral inorgânico ${ }^{1}$ & 0,05 & - & - & - \\
\hline $\begin{array}{l}\text { Suplemento mineral e vitamínico e } \\
\text { aminoácidos }^{2}\end{array}$ & 3,00 & 3,00 & 3,00 & 3,00 \\
\hline Caulim & 0,1000 & 0,1048 & 0,0596 & 0,0144 \\
\hline Selênio levedura ${ }^{3}$ & - & 0,0100 & 0,0200 & 0,0300 \\
\hline Proteinato de zinco ${ }^{4}$ & - & 0,0133 & 0,0266 & 0,0399 \\
\hline Proteinato de manganês ${ }^{5}$ & - & 0,0133 & 0,0266 & 0,0399 \\
\hline Proteinato de ferro ${ }^{6}$ & - & 0,0066 & 0,0132 & 0,0198 \\
\hline Proteinato de cobre ${ }^{7}$ & - & 0,0020 & 0,0040 & 0,0060 \\
\hline Total & 100,00 & 100,00 & 100,00 & 100,00 \\
\hline \multicolumn{5}{|l|}{ Nível nutricional calculado } \\
\hline Energia metabolizável (kcal/kg) & 2700 & 2700 & 2700 & 2700 \\
\hline Proteína bruta (\%) & 16,000 & 16,000 & 16,000 & 16,000 \\
\hline Cálcio (\%) & 4,050 & 4,050 & 4,050 & 4,050 \\
\hline Fósforo disponível (\%) & 0,381 & 0,381 & 0,381 & 0,381 \\
\hline Metionina total $(\%)$ & 0,320 & 0,320 & 0,320 & 0,320 \\
\hline Lisina total $(\%)$ & 0,853 & 0,853 & 0,853 & 0,853 \\
\hline Extrato etéreo (\%) & 2,739 & 2,739 & 2,739 & 2,739 \\
\hline Fibra bruta (\%) & 2,946 & 2,946 & 2,946 & 2,946 \\
\hline Sódio total (\%) & 0,180 & 0,180 & 0,180 & 0,180 \\
\hline
\end{tabular}

$\mathrm{T}_{1}$ : dieta basal $+0,3 \mathrm{ppm}$ de selenito de $\mathrm{Na}+60 \mathrm{ppm}$ óxido de $\mathrm{Zn}+60 \mathrm{ppm}$ monóxido de $\mathrm{Mn}+30 \mathrm{ppm}$ de sulfato $\mathrm{Fe}+0,5 \mathrm{ppm}$ de iodato de $\mathrm{Ca}+6,0 \mathrm{ppm}$ de sulfato de $\mathrm{Cu} ; \mathrm{T}_{2}$ : dieta basal $+0,1 \mathrm{ppm}$ de Se levedura $+20 \mathrm{ppm}$ de proteinato de $\mathrm{Zn}+20 \mathrm{ppm}$ de proteinato de $\mathrm{Mn}+10 \mathrm{ppm}$ de proteinato de $\mathrm{Fe}+2,0 \mathrm{ppm}$ de proteinato de $\mathrm{Cu}+0,5 \mathrm{ppm}$ de iodato de $\mathrm{Ca} ; \mathrm{T}_{3}$ : dieta basal $+0,2 \mathrm{ppm}$ de Se levedura $+40 \mathrm{ppm}$ de proteinato de $\mathrm{Zn}+40 \mathrm{ppm}$ de proteinato de $\mathrm{Mn}+20 \mathrm{ppm}$ de proteinato de $\mathrm{Fe}+4,0 \mathrm{ppm}$ de proteinato de $\mathrm{Cu}$ $+0,5 \mathrm{ppm}$ de iodato de $\mathrm{Ca} ; \mathrm{T}_{4}$ : dieta basal $+0,3 \mathrm{ppm}$ de Se levedura $+60 \mathrm{ppm}$ de proteinato de $\mathrm{Zn}+60 \mathrm{ppm}$ de proteinato de $\mathrm{Mn}+$ 30ppm de proteinato de $\mathrm{Fe}+6,0 \mathrm{ppm}$ de proteinato de $\mathrm{Cu}+0,5 \mathrm{ppm}$ de iodato de $\mathrm{Ca}$.

Garantia por $\mathrm{kg}$ do produto:

${ }^{1}$ Suplemento mineral inorgânico: monóxido de Mn: 140000mg, óxido de Zn: $100000 \mathrm{mg}$, sulfato ferroso: $120000 \mathrm{mg}$, sulfato de Cu: $200000 \mathrm{mg}$, iodato de Ca: $1400 \mathrm{mg}$, selenito de Na: $700 \mathrm{mg}$.

${ }^{2}$ Suplemento mineral, vitamínico e aminoácido: Ca: 269g, P: 94g, Mn: 2334mg, Zn: 1667mg, Fe: 2000mg, Cu: 334mg, I: 12mg, Se: $10,2 \mathrm{mg}$, vit. A: $334000 \mathrm{UI}$, vit. $\mathrm{D}_{3}: 67000 \mathrm{UI}$, vit. E: $234 \mathrm{mg}$, vit. $\mathrm{K}_{3}: 50 \mathrm{mg}$, vit. $\mathrm{B}_{1}: 54 \mathrm{mg}$, vit. $\mathrm{B}_{2} 147 \mathrm{mg}$, vit. $\mathrm{B}_{6}: 100 \mathrm{~g}$, vit. $\mathrm{B}_{12}$ : $400 \mathrm{mcg}$, niacina: $867 \mathrm{mg}$, ácido fólico: $24 \mathrm{mg}$, ácido pantotênico: $334 \mathrm{mg}$, metionina: $34 \mathrm{~g}$.

${ }^{3}$ Suplemento mineral orgânico: Se levedura: $1000 \mathrm{mg} / \mathrm{kg}$.

${ }^{4}$ Suplemento mineral orgânico: proteinato de $\mathrm{Zn}: 15 \%$.

${ }^{5}$ Suplemento mineral orgânico: proteinato de $\mathrm{Mn}: 150000 \mathrm{mg} / \mathrm{kg}$.

${ }^{6}$ Suplemento mineral orgânico: proteinato de Fe: $150000 \mathrm{~g}$.

${ }^{7}$ Suplemento mineral orgânico: proteinato de $\mathrm{Cu}: 100000 \mathrm{~g}$.

As variáveis de qualidade externa dos ovos analisadas foram peso do ovo, gravidade específica, peso e espessura da casca, e as de qualidade interna foram altura do albúmen, unidade Haugh e pesos da gema e do albúmen do ovo. Essas variáveis foram analisadas para cada período de 28 dias, e a produção de ovos teve controle diário.

Ao final do período experimental, foram abatidas três aves de cada tratamento, para coleta dos ossos da tíbia, esquerdo e direito. Os ossos foram mantidos sob refrigeração e, posteriormente, foram encaminhados para a análise de resistência óssea, utilizando-se o equipamento Instron Universal Testing Machine (MOD. 1130). Para a obtenção da medida de resistência dos ossos da tíbia, foi utilizada uma célula larga, plana, de pistão chato com diâmetro de $24 \mathrm{~mm}$ e com calibre de $50 \mathrm{~kg}$, e a velocidade da carga e da cabeça empregada para a leitura dos resultados foi de $10 \mathrm{~cm} / \mathrm{min}$. 
O delineamento experimental utilizado foi o inteiramente ao acaso, e os dados foram submetidos à análise de variância a $5 \%$ de probabilidade, regressão polinomial e comparação de médias por meio de contrastes polinomiais.

\section{RESULTADOS E DISCUSSÃO}

Os resultados das variáveis de qualidade externa dos ovos estão apresentados na Tab. 3. O peso do ovo produzido pelas poedeiras que receberam $\mathrm{T}_{3}$ - dieta suplementada com percentual intermediário de minerais orgânicos - apresentou significativamente $(\mathrm{P}=0,0234)$ valores mais elevados. O resultado assemelha-se ao de Xavier et al. (2004), que observaram ovos mais pesados $(1,3 \%)$ por aves alimentadas com minerais quelatados. O maior peso do ovo é reflexo do aumento da gema, do albúmen e da casca do ovo (Rutz et al., 2003), logo proveniente do melhor aproveitamento de nutrientes. Araujo et al. (2008) afirmaram que, além da quantidade e da qualidade dos minerais na ração, a inter-relação entre os minerais e os demais nutrientes também é importante.

Acompanhando o aumento no peso do ovo, as aves alimentadas com $\mathrm{T}_{3}$ produziram ovos com cascas mais pesadas, apresentando as duas variáveis, peso do ovo (PO) e de casca (PSCS), pela análise de regressão polinomial, resposta quadrática: $\mathrm{PO}=32,968+19,116$ minerais orgânicos - 3,107 minerais orgânicos ${ }^{2}$; e PSCS = $4,057+1,480$ minerais orgânicos - 0,237 minerais orgânicos ${ }^{2}$. Lundeen (2001) observou melhora da qualidade de casca de ovos de poedeiras quando suplementadas com manganês e zinco quelatados no período de 20 a 60 semanas de idade. O Zn participa, sob a forma de carbonato, da fixação do $\mathrm{Ca}$ nos ossos e da ativação de sistemas enzimáticos, entre outras funções orgânicas (Torres, 1969). A adição de zinco metionina à dieta, comparada com óxido de zinco para aves de postura, provoca uma redução nos problemas de casca do ovo, por aumentar a resistência à quebra e a atividade da anidrase carbônica, principal enzima responsável pela síntese de carbonato de cálcio para a formação da casca do ovo.

Para gravidade específica (GE) e espessura da casca do ovo (ESC), não foram observadas diferenças significativas $(\mathrm{P}>0,05)$ entre os tratamentos, e a resposta polinomial verificada foi constante: $\mathrm{GE}=1092 ; \mathrm{ECS}=41,98$. Dale e Strong (1998), ao estudarem a influência de um complexo mineral orgânico na gravidade específica dos ovos, também não encontraram diferença entre os tratamentos utilizados, mas, quando foi analisado um período em que foram removidos os minerais orgânicos, os autores observaram menor valor de gravidade específica para os ovos das aves que receberam o mineral orgânico quando comparados aos das aves que receberam minerais na forma inorgânica. Contudo, no presente experimento, a qualidade da casca dos ovos, representada pela gravidade específica, mesmo não diferindo entre os tratamentos, apresentou valor acima de 1080, valor mínimo para que os ovos comerciais resistam ao transporte e ao processamento (Balander et al., 1997).

Contrastando os tratamentos com mineral inorgânico $\left(\mathrm{T}_{1}\right)$ e minerais orgânicos no maior percentual $\left(\mathrm{T}_{4}\right)$, observou-se que as aves alimentadas com os minerais inorgânicos produziram ovos mais pesados $(\mathrm{P}<0,0001)$. Portanto, não é conveniente utilizar os mesmos níveis de minerais orgânicos em substituição aos inorgânicos, pois tanto a deficiência como o excesso de minerais na dieta podem proporcionar desequilíbrio mineral, prejudicando a absorção de nutrientes e o desempenho animal (Araujo et al., 2008).

$\mathrm{Na}$ Tab. 4, podem ser observados os resultados das variáveis de qualidade interna dos ovos estudadas. Para as variáveis altura do albúmen, unidade Haugh e peso da gema, não foram verificados efeitos significativos $(\mathrm{P}>0,05)$ dos tratamentos. Estes dados assemelham-se aos obtidos por Lundeen (2001) e Saldanha et al. (2009), que não observaram efeito de tratamento sobre a qualidade interna de ovos, ao contrastarem dietas que continham minerais inorgânicos com dietas que apresentavam na sua formulação minerais orgânicos.

Observou-se efeito significativo $(\mathrm{P}=0,0375)$ dos tratamentos sobre o peso do albúmen, o qual apresentou maior valor para os ovos das aves alimentadas com o $\mathrm{T}_{3}$. Também se verificou resposta polinomial quadrática para pesos da gema e do albúmen. Embora não significativo, o peso da gema foi maior para os ovos produzidos pelas aves que receberam o $\mathrm{T}_{3}$. Portanto, o observado associa-se ao maior peso do ovo proveniente também desse tratamento. 
Qualidade de ovos...

Tabela 3. Qualidade externa de ovos de poedeiras alimentadas com minerais orgânicos, no período de 30 a 70 semanas de idade

\begin{tabular}{|c|c|c|c|c|}
\hline Tratamento $^{1}$ & $\begin{array}{l}\text { Peso do ovo } \\
\text { (g) }\end{array}$ & $\begin{array}{l}\text { Gravidade } \\
\text { específica }\end{array}$ & $\begin{array}{l}\text { Peso da casca do } \\
\text { ovo }(\mathrm{g})\end{array}$ & $\begin{array}{c}\text { Espessura da } \\
\text { casca do ovo } \\
(\mathrm{mm})\end{array}$ \\
\hline $\mathrm{T}_{1}$ & 60,80 & 1092 & 6,18 & 0,42 \\
\hline $\mathrm{T}_{2}$ & 59,36 & 1092 & 6,15 & 0,42 \\
\hline $\mathrm{T}_{3}$ & 61,48 & 1092 & 6,29 & 0,42 \\
\hline $\mathrm{T}_{4}$ & 59,08 & 1093 & 6,13 & 0,42 \\
\hline $\mathrm{P}^{2}$ & 0,0234 & 0,5888 & 0,3002 & 0,1793 \\
\hline Coeficiente de variação, $\%$ & 10,19 & 0,19 & 4,22 & 2,62 \\
\hline $\begin{array}{l}\text { Componente polinomial de maior } \\
\text { grau significativo }\end{array}$ & 2 & 0 & 2 & 0 \\
\hline Curva ajustada ${ }^{3}$ & Quadrática & Constante & Quadrática & Constante \\
\hline Coeficiente de determinação $\left(\mathrm{R}^{2}\right)$ & 0,99 & 0 & 0,99 & 0 \\
\hline \multicolumn{5}{|l|}{ Contraste polinomial } \\
\hline $\begin{array}{l}\text { Inorgânicos }\left(\mathrm{T}_{1}\right) \text { versus orgânicos } \\
\text { no maior nível }\left(\mathrm{T}_{4}\right)\end{array}$ & $<0,0001$ & 0,0605 & 0,3746 & 0,8970 \\
\hline $\begin{array}{l}\text { Inorgânicos }\left(\mathrm{T}_{1}\right) \text { versus orgânicos } \\
\left(\mathrm{T}_{2}, \mathrm{~T}_{3}, \mathrm{~T}_{4}\right)\end{array}$ & 0,0879 & 0,1803 & 0,8596 & 0,1510 \\
\hline
\end{tabular}

${ }^{1}$ Tratamento: $\mathrm{T}^{\mathrm{I}}$ : dieta basal + 0,3ppm de selenito de $\mathrm{Na}+60 \mathrm{ppm}$ óxido de $\mathrm{Zn}+60 \mathrm{ppm}$ monóxido de $\mathrm{Mn}+30 \mathrm{ppm}$ de sulfato $\mathrm{Fe}+$ $0,5 \mathrm{ppm}$ de iodato de $\mathrm{Ca}+6,0 \mathrm{ppm}$ de sulfato de $\mathrm{Cu} ; \mathrm{T}_{2}$ : dieta basal $+0,1 \mathrm{ppm}$ de Se levedura $+20 \mathrm{ppm}$ de proteinato de $\mathrm{Zn}+20 \mathrm{ppm}$ de proteinato de $\mathrm{Mn}+10 \mathrm{ppm}$ de proteinato de $\mathrm{Fe}+2,0 \mathrm{ppm}$ de proteinato de $\mathrm{Cu}+0,5 \mathrm{ppm}$ de iodato de $\mathrm{Ca}$; $\mathrm{T}_{3}$ : dieta basal $+0,2 \mathrm{ppm}$ de Se levedura $+40 \mathrm{ppm}$ de proteinato de $\mathrm{Zn}+40 \mathrm{ppm}$ de proteinato de $\mathrm{Mn}+20 \mathrm{ppm}$ de proteinato de $\mathrm{Fe}+4,0 \mathrm{ppm}$ de proteinato de $\mathrm{Cu}+0,5 \mathrm{ppm}$ de iodato de $\mathrm{Ca} ; \mathrm{T}_{4}$ : dieta basal $+0,3 \mathrm{ppm}$ de Se levedura $+60 \mathrm{ppm}$ de proteinato de $\mathrm{Zn}+60 \mathrm{ppm}$ de proteinato de $\mathrm{Mn}+$ 30ppm de proteinato de $\mathrm{Fe}+6,0 \mathrm{ppm}$ de proteinato de $\mathrm{Cu}+0,5 \mathrm{ppm}$ de iodato de $\mathrm{Ca}$.

${ }^{2} \mathrm{P}$ : probabilidade de declarar significativo efeito inexistente dos minerais.

${ }^{3}$ Equação da função polinomial ajustada: peso do ovo $=32,968+19,116$ minerais orgânicos $-3,107$ minerais orgânicos ${ }^{2}$; gravidade específica $=1092$; peso da casca do ovo $=4,057+1,480$ minerais orgânicos $-0,237$ minerais orgânicos ${ }^{2}$; espessura da casca do ovo $=0,42$.

Ao contrastar os tratamentos 1 e 4 , verificou-se efeito significativo para pesos da gema $(\mathrm{P}=0,0036)$ e do albúmen $(\mathrm{P}=0,0011)$, sendo os maiores pesos para os ovos das poedeiras alimentadas com o $\mathrm{T}_{1}$. Ressalta-se que a utilização dos minerais orgânicos em substituição aos inorgânicos não deve ser realizada nos mesmos percentuais (Araujo et al., 2008).

Segundo Booden (1986) e Santos (2005), o albúmen constitui cerca de $60 \%$, e a gema $30 \%$ do peso do ovo, logo gema e albúmen mais pesados refletem em ovos mais pesados. Utilizando uma combinação de minerais orgânicos - zinco, selênio e manganês - Rutz et al. (2003) observaram melhora no peso do albúmen e da gema do ovo de poedeiras, durante o segundo ciclo de postura. Um albúmen mais consistente pode ser reflexo da utilização de selênio orgânico (Wakebe, 1999) ou devido a uma combinação de minerais orgânicos selênio, zinco e manganês (Rutz et al., 2003). Ainda, segundo Surai (2006), citado por Rutz e Murphy (2009), a suplementação das dietas de poedeiras com selênio orgânico, entre outros benefícios, resulta em maior concentração deste mineral em todas as partes do ovo.

Não se observou efeito significativo dos tratamentos para a variável resistência óssea (ROS), obtendo-se resposta polinomial constante ( $\mathrm{ROS}=14,28 \mathrm{~kg})$, conforme pode ser observado na Tab. 5. Contudo, algumas pesquisas (Henry et al., 1992; Wedekind et al., 1992; Brito et al., 2006) demonstraram haver resultados significativos para esta variável quando a dieta dos animais foi suplementada com minerais orgânicos. 
Tabela 4. Qualidade interna dos ovos de poedeiras alimentadas com minerais orgânicos, no período de 30 a 70 semanas de idade

\begin{tabular}{|c|c|c|c|c|}
\hline Tratamento $^{1}$ & $\begin{array}{l}\text { Altura do } \\
\text { albúmen } \\
(\mathrm{mm})\end{array}$ & $\begin{array}{l}\text { Unidade } \\
\text { Haugh }\end{array}$ & $\begin{array}{c}\text { Peso da gema } \\
\text { (g) }\end{array}$ & $\begin{array}{c}\text { Peso do } \\
\text { albúmen (g) }\end{array}$ \\
\hline $\mathrm{T}_{1}$ & 7,39 & 85,29 & 15,01 & 36,70 \\
\hline $\mathrm{T}_{2}$ & 7,16 & 84,26 & 14,87 & 35,66 \\
\hline $\mathrm{T}_{3}$ & 7,35 & 84,61 & 15,25 & 36,88 \\
\hline $\mathrm{T}_{4}$ & 7,21 & 84,65 & 14,57 & 35,64 \\
\hline $\mathrm{P}^{2}$ & 0,4586 & 0,8456 & 0,1924 & 0,0375 \\
\hline Coeficiente de variação, $\%$ & 11,81 & 6,41 & 6,54 & 5,75 \\
\hline $\begin{array}{l}\text { Componente polinomial de maior grau } \\
\text { significativo } \\
\text { Curva ajustada } \\
\text { Coeficiente de determinação }\left(\mathrm{R}^{2}\right) \\
\end{array}$ & $\begin{array}{c}0 \\
\text { Constante } \\
0 \\
\end{array}$ & $\begin{array}{c}0 \\
\text { Constante } \\
0 \\
\end{array}$ & $\begin{array}{c}2 \\
\text { Quadrática } \\
0,89\end{array}$ & $\begin{array}{c}2 \\
\text { Quadrática } \\
0,95\end{array}$ \\
\hline \multicolumn{5}{|l|}{ Contraste polinomial } \\
\hline $\begin{array}{l}\text { Inorgânicos }\left(T_{1}\right) \text { versus orgânicos no maior } \\
\text { nível }\left(T_{4}\right) \\
\text { Inorgânicos }\left(T_{1}\right) \text { versus orgânicos }\left(T_{2}, T_{3} \text {, }\right. \\
\left.T_{4}\right)\end{array}$ & 0,1658 & 0,4440 & 0,0036 & 0,0011 \\
\hline \multicolumn{5}{|c|}{$\begin{array}{l}{ }^{1} \text { Tratamento: } \mathrm{T}^{1}: \text { dieta basal }+0,3 \mathrm{ppm} \text { de selenito de } \mathrm{Na}+60 \mathrm{ppm} \text { óxido de } \mathrm{Zn}+60 \mathrm{ppm} \text { monóxido de } \mathrm{Mn}+30 \mathrm{ppm} \text { de sulfato } \mathrm{Fe} \\
+0,5 \mathrm{ppm} \text { de iodato de } \mathrm{Ca}+6,0 \mathrm{ppm} \text { de sulfato de } \mathrm{Cu} \text {; } \mathrm{T}_{2} \text { : dieta basal }+0,1 \mathrm{ppm} \text { de } \mathrm{Se} \text { levedura }+20 \mathrm{ppm} \text { de proteinato de } \mathrm{Zn}+ \\
\text { 20ppm de proteinato de } \mathrm{Mn}+10 \mathrm{ppm} \text { de proteinato de } \mathrm{Fe}+2,0 \mathrm{ppm} \text { de proteinato de } \mathrm{Cu}+0,5 \mathrm{ppm} \text { de iodato de } \mathrm{Ca} \text {; } \mathrm{T}_{3}: \text { dieta basal } \\
+0,2 \mathrm{ppm} \text { de } \mathrm{Se} \text { levedura }+40 \mathrm{ppm} \text { de proteinato de } \mathrm{Zn}+40 \mathrm{ppm} \text { de proteinato de } \mathrm{Mn}+20 \mathrm{ppm} \text { de proteinato de } \mathrm{Fe}+4,0 \mathrm{ppm} \text { de } \\
\text { proteinato de } \mathrm{Cu}+0,5 \mathrm{ppm} \text { de iodato de } \mathrm{Ca} \text {; } \mathrm{T}_{4} \text { : dieta basal }+0,3 \mathrm{ppm} \text { de } \mathrm{Se} \text { levedura }+60 \mathrm{ppm} \text { de proteinato de } \mathrm{Zn}+60 \mathrm{ppm} \text { de } \\
\text { proteinato de } \mathrm{Mn}+30 \mathrm{ppm} \text { de proteinato de } \mathrm{Fe}+6,0 \mathrm{ppm} \text { de proteinato de } \mathrm{Cu}+0,5 \mathrm{ppm} \text { de iodato de Ca. } \\
{ }^{2} \mathrm{P} \text { : probabilidade de declarar significativo efeito inexistente dos minerais. } \\
{ }^{3} \text { Equação da função polinomial ajustada: altura do albúmen }=7,28 \text {; unidade Haugh }=84,78 \text {; peso da gema }=7,295+5,801 \\
\text { minerais orgânicos }-0,972 \text { minerais orgânicos }{ }^{2} ; \text { peso do albúmen }=18,520+12,247 \text { minerais orgânicos }-1,992 \text { minerais } \\
\text { orgânicos }^{2} \text {. }\end{array}$} \\
\hline
\end{tabular}

Tabela 5. Resistência dos ossos da tíbia de poedeiras alimentadas com minerais orgânicos, no período de 30 a 70 semanas de idade

\begin{tabular}{lc} 
Tratamento $^{1}$ & Resistência óssea (kg) \\
\hline $\mathrm{T}_{1}$ & 14,13 \\
$\mathrm{~T}_{2}$ & 14,07 \\
$\mathrm{~T}_{3}$ & 14,73 \\
$\mathrm{~T}_{4}$ & 14,20 \\
\hline Média & 14,28 \\
\hline $\mathrm{P}^{2}$ & 0,9527 \\
\hline Coeficiente de variação, \% & 11,25 \\
Componente polinomial de maior grau significativo & 0 \\
Curva ajustada & Constante \\
Coeficiente de determinação $\left(\mathrm{R}^{2}\right)$ & 0 \\
\hline Contraste polinomial & 0,9578 \\
\hline Inorgânicos $\left(\mathrm{T}_{1}\right)$ versus orgânicos no maior nível $\left(\mathrm{T}_{4}\right)$ & 0,8544 \\
Inorgânicos $\left(\mathrm{T}_{1}\right)$ versus orgânicos $\left(\mathrm{T}_{2}, \mathrm{~T}_{3}, \mathrm{~T}_{4}\right)$ & \\
\hline
\end{tabular}

${ }^{\mathrm{I}}$ Tratamento: $\mathrm{T}^{1}$ : dieta basal + 0,3ppm de selenito de $\mathrm{Na}+60 \mathrm{ppm}$ óxido de $\mathrm{Zn}+60 \mathrm{ppm}$ monóxido de $\mathrm{Mn}+30 \mathrm{ppm}$ de sulfato $\mathrm{Fe}+0,5 \mathrm{ppm}$ de iodato de $\mathrm{Ca}+6,0 \mathrm{ppm}$ de sulfato de $\mathrm{Cu} ; \mathrm{T}_{2}$ : dieta basal $+0,1 \mathrm{ppm}$ de $\mathrm{Se}$ levedura + $20 \mathrm{ppm}$ de proteinato de $\mathrm{Zn}+20 \mathrm{ppm}$ de proteinato de $\mathrm{Mn}+10 \mathrm{ppm}$ de proteinato de $\mathrm{Fe}+2,0 \mathrm{ppm}$ de proteinato de $\mathrm{Cu}+0,5 \mathrm{ppm}$ de iodato de $\mathrm{Ca} ; \mathrm{T}_{3}$ : dieta basal $+0,2 \mathrm{ppm}$ de Se levedura $+40 \mathrm{ppm}$ de proteinato de $\mathrm{Zn}+40 \mathrm{ppm}$ de proteinato de $\mathrm{Mn}+20 \mathrm{ppm}$ de proteinato de $\mathrm{Fe}+4,0 \mathrm{ppm}$ de proteinato de $\mathrm{Cu}+0,5 \mathrm{ppm}$ de iodato de $\mathrm{Ca} ; \mathrm{T}_{4}$ : dieta basal $+0,3 \mathrm{ppm}$ de Se levedura $+60 \mathrm{ppm}$ de proteinato de $\mathrm{Zn}+60 \mathrm{ppm}$ de proteinato de $\mathrm{Mn}+30 \mathrm{ppm}$ de proteinato de $\mathrm{Fe}+6,0 \mathrm{ppm}$ de proteinato de $\mathrm{Cu}+0,5 \mathrm{ppm}$ de iodato de $\mathrm{Ca}$.

${ }^{2} \mathrm{P}$ : probabilidade de declarar significativo efeito inexistente dos minerais.

${ }^{3}$ Equação da função polinomial ajustada: resistência óssea $=14,28 \mathrm{~kg}$. 
Os minerais orgânicos utilizados, no presente estudo, foram adequadamente absorvidos e metabolizados. Parte disso pode, possivelmente, ser justificado pela boa formação óssea observada. O osso é um tipo de tecido conjuntivo formado por matriz orgânica, onde microminerais (cobre, manganês e zinco), como cofatores enzimáticos, apresentam papel fundamental na sua síntese e uma porção mineral, constituída de fosfato de cálcio. O fornecimento adequado de cálcio na dieta, por exemplo, não propicia carência deste mineral para a formação da casca do ovo, portanto não há necessidade da utilização de cálcio do osso medular (Saldanha et al., 2009). Desse modo, as aves depositam as quantidades necessárias de minerais nos ossos, que os tornam mais densos e resistentes, mesmo com o aumento da idade (Paz, 2006). Não havendo perdas na soma total de minerais da estrutura óssea, não ocorre aumento na fragilidade e susceptibilidade a fraturas, principalmente da tíbia (Whitehead e Fleming, 2000).

\section{CONCLUSÕES}

O fornecimento de dietas suplementadas com minerais orgânicos, em nível intermediário de substituição dos minerais inorgânicos, para poedeiras semipesadas, resulta na produção de ovos mais pesados, com maior peso de albúmen, e na manutenção das demais características de qualidade externa e interna dos ovos e de resistência óssea.

\section{REFERÊNCIAS}

ARAUJO, J.A.; SILVA, J.H.V.S.; AMÂNCIO, A.L.L. et al. Fontes de minerais para poedeiras. Acta Vet. Bras., v.2, p.53-60, 2008.

BALANDER, R.J.; FLEGAL, C.J.; STEFTON, $\mathrm{T}$. The effects of SSF on egg production and egg specific gravity in laying hens. Poult. Sci., v.76, p.3, 1997.

BONDEI, A.A. (Ed.) Animal nutrition. New York: Wiley, 1987. 540p.

BOODEN, M. The egg - big things in a small package. Food Scie. Newsletter. Publication of Hazleton Laboratories for the food and feed industries. v.13, 1986.
BRITO, J.A.G.; BERTECHINI, A.G.; FASSANI, É.J. et al. Uso de microminerais sob a forma de complexo orgânico em rações para frangas de reposição no período de 7 a 12 semanas de idade. Rev. Bras. Zootec. v.35, p.1342-1348, 2006.

CLYDESDALE, F.M. Mineral interactions in foods. In: BODWELL, C.E.; ERDMAN Jr., J.W. (Eds.). Nutrient interactions. New York: Marcel Dekker, 1998. p.257-268.

DALE, N.; STONG, C.F. Inability to demonstrate na effect of eggshell 49 on shell quality in older laying hens. J. Appl. Poult. Sci., v.7, p.219-224, 1998.

HENRY, P.R.; AMMERMAN, C.B.; LITTELL, R.C. Relative bioavailability of manganese from a manganese-methionine complex and inorganic sources for ruminants. J. Dairy Sci., v.75, p.3473-3478, 1992.

JUNQUEIRA O.M. Nutrição animal: Quelatos na alimentação animal - Boletim técnico. 2008. Disponível em: <http://www.pedrovet.com.br/trabalhosC/Quelat osnaAlimentacao.doc.>. Acessado em: 05 jun. 2011.

KIENHOLZ, E.W. Zinc methionine for stressed laying hens. Poult. Sci., v.71, p.829-832, 1992.

KRATZER, F.H.; VOHRA, P. (Eds.). Chetales in nutrition. Boca Raton: CRC, 1986. p.5-33.

LUNDEEN, T. Mineral proteinates may have positive effect on shell quality. Feedstuffs, v.73, p.10-15, 2001.

MALLETO, S. Allá riscoperta dei mineralli. Inf. Zootec., v.44, p.51-84, 1997.

McDOWELL, L.R. Minerals in animal and human nutrition. San Diego: Academic, 1992. $524 \mathrm{p}$.

PAZ, I.C.L.A. Acompanhamento do desenvolvimento do tecido ósseo de matrizes pesadas por meio da técnica de densitometria óptica em imagens radiográficas, qualidade óssea e produção de ovos. 2006. 100f. Tese (Doutorado em Zootecnia) - Curso de Zootecnia, Universidade Estadual Paulista, Botucatu, SP.

POLLI, S.R. Minerais orgânicos na alimentação de cães e gatos. Bol. Inf. Nutron Pet, v.1, p.3, 2002. 
REDDY, A.B.; DWIVED, J.N.; ASHMEAD, A.D. Mineral chelation generates profit. MissetWorld Poult., v.8, p.13-15, 1992.

RUTZ, F.; ANCIUTI, M.A.; RECH, J.L. et al. Following response to Sel-Plex ${ }^{\circledR}$ and other organic minerals trough the broiler breeder maze: case studies in Brazil. In: PROCEEDINGS OF ALLTECH'S $21^{\text {st }}$ ANNUAL SYMPOSIUM, 21., 2005, Nottinghan. Anais... Nottingham, 2005. p.502-513. (Resumo).

RUTZ, F.; PAN, E.A.; XAVIER, E.G. et al. Meeting selenium demands of modern poultry: responses to Sel-Plex ${ }^{\mathrm{TM}}$ organic selenium in broiler and breeder diets. In: PROCEEDINGS OF ALLTECH'S $19^{\text {th }}$ ANNUAL SYMPOSIUM, 19., 2003, Nottinghan. Anais... Nottingham, 2003. p.147-161. (Resumo).

RUTZ, F.; MURPHY, R. Minerais orgânicos para aves e suínos. In: CONGRESSO INTERNACIONAL SOBRE USO DA LEVEDURA NA ALIMENTAÇÃO ANIMAL, 1., 2009, Campinas, SP. Anais ... Campinas: CBNA, 2009. p.21-36. Disponível em: <www.avisite.com.br/cet/img/20091103_minerai s.pdf>. Acessado em: 7 jun. 2011.

SALDANHA, E.S.P.B.; GARCIA, E.A.; PIZZOLANTE, E.A. et al. Effect of organic mineral supplementation on the egg quality of semi-heavy layers in their second cycle of lay. Braz. J. Poult. Sci., v.11, p.215-222, 2009.

SANTOS, M.S.V. Avaliação do desempenho $e$ qualidade dos ovos de poedeiras comerciais, submetidas às dietas suplementadas com diferentes óleos vegetais. 2005. 174f. Tese (Doutorado em Zootecnia) - Universidade Federal do Ceará, Fortaleza.

SECHINATTO, A.S. Efeito da suplementação dietética com micro minerais orgânicos na produção e qualidade dos ovos de galinhas poedeiras. 2003. 61f. Dissertação. (Mestrado em Medicina Veterinária) - Faculdade de Medicina Veterinária e Zootecnia da Universidade de São Paulo, Pirassununga, SP.
TORRES, A.P. (Ed.). Alimentação das aves. São Paulo: Edições Melhoramentos, 1969. 259p.

UNIÃO Brasileira de Avicultura (UBA). Protocolo de bem-estar para aves poedeiras, 2008. Disponível em: <http://www.abef.com.br/uba/arquivos/protocolo _de_bem_estar_para_aves_poedeiras_final_11_0 7_08.pdf>. Acessado em: 29 mai. 2011.

VEIGA, J.B.; CARDOSO, E.C. Criação de gado leiteiro na zona Bragantina, 2005. Disponível em:

<http://sistemasdeproducao.cnptia.embrapa.br/Fo ntesHTML/Leite/GadoLeiteiroZonaBragantina/p aginas/apresentacao.htm>. Acessado em: 29 mai. 2011

WAKEBE, M. Organic selenium and egg freshness. In: PROCEEDINGS OF ALLTECH'S FIFTEENTH ANNUAL SYMPOSIUM, 15., 1999, Nottinghan. Anais ... Nottingham, 1999. p.595. (Resumo).

WEDEKIND, K.J.; HORTIN, A.E.; BAKER, D.H. Methodology for assessing zinc bioavailability: efficacy estimative for zinc-methionine, zinc sulfate and zinc oxidase. J. Anim. Sci., v.70, p.178-187, 1992.

WHITEHEAD, C.C.; FLEMING, R.H. Osteoporosis in cage layers. Poult. Sci., v.7, p.1033-1041. 2000.

XAVIER, G.B.; RUTZ, F.; DIONELLO, N.J.L. et al. Desempenho de poedeiras alimentadas com dietas contendo selênio, zinco e manganês orgânico durante o segundo ciclo de produção. In: RONDA LATINO-AMERICANA DA ALLTECH, 14., SIMPÓSIO DA INDÚSTRIA DE ALIMENTOS ANIMAL, 2004, Kentucky, Lexington. Anais... Kentucky, Lexington, 2004. p.8. (Resumo). 